\title{
On delayed loss of stability in one mechanical problem
}

\author{
E.V. Shchetinina ${ }^{1}$ \\ ${ }^{1}$ Samara National Research University, 34 Moskovskoe Shosse, 443086, Samara, Russia
}

\begin{abstract}
Consider a double pendulum under the external force. This model can be described by the multi-speed systems of ordinary differential equations. We study the existence of stable motions depending on the parameters of the system and on the value of the external force. Critical values of the parameters are defined.
\end{abstract}

Ключевые слова: slow-fast systems; delayed loss of stability; double pendulum

\section{Introduction}

Numerical investigation of dynamical systems can be very complicated due to the high dimensions of systems and presence of big and small parameters. In such cases it is reasonable to use combination of numerical and analytical methods. By analytical methods we can decrease the dimension of the model, restrict the domain of the investigation, define the different types of behavior depending on the parameters values. And then by using numerical methods we are able to investigate specific solutions and manifolds of them.

One such analytical method is the method of the integral manifolds. With the help of it we are able to decrease the number of variables, to restrict the domains of the investigation, to predict different types of behaviour of the solutions to the system.

In this paper we consider a double pendulum with elastic hinges under the external force. We assume that the force is slowly changing in time. Our goal is to investigate the behavior of the solutions for different values of the parameters of the system and to find the influence of the force growth to the pendulum motions.

\section{Integral manifolds}

Integral manifolds method is an efficient tool for studying complicated dynamical systems. It was developed by many authors. This method is quiet successful for studying multi-scaled systems (see, e.g. [3]). The main ideas if this method are as follows.

Consider the singularly perturbed system:

$$
\frac{d x}{d t}=f(x, y, a, \varepsilon), \varepsilon \frac{d y}{d t}=g(x, y, a, \varepsilon),
$$

with $x \in R^{n}, \mathrm{y} \in R^{m}, \mathrm{t} \in R, a$ is a parameter, $0<\varepsilon \ll 1$. Here $x$ is a slow variable, $y$ is a fast variable. Integral manifold of such a system is an invariant set of the system. We are interested in the integral manifolds of the form $y=h(x, \varepsilon)$, with $h$ smoothly depending on $\varepsilon$. This type of manifolds is called slow integral manifolds. The motion on this manifolds is provided by the equation $\dot{x}=\mathrm{f}(\mathrm{x}, \mathrm{h}(\mathrm{x}, \varepsilon), \varepsilon)$.

Suppose that the degenerated equation $g(x, y, a, 0)=0$ has an isolated root $y=h_{0}(x, a)$. The surface defined by the relation $y=h_{0}(x, a)$ is called slow manifold. Consider the Jacobi matrix $\partial g / \partial y(x, \mathrm{y}, a)$ with $y=h_{0}(x, a)$. If all eigenvalues of the Jacobi matrix are in the left open complex half-plane, then the slow manifold is attractive. If there exists at least one eigenvalue in the right complex half-plane, then the slow manifold is repelling. In the $\varepsilon$-neighbourhoods of the attracting and repelling slow manifolds there exist attracting and repelling slow integral manifolds.

The surface with the condition that the eigenvalues of the Jacobi matrix are on the imaginary axis is called the surface of change of attractivity. Due to the presence of the additional parameter $a$ we are able to glue together attracting and repelling slow integral manifolds at one point. Thus we obtain the solution that follows first an attractive slow manifold and then repelling slow manifold. Such a solution is called a canard solution.

Also there exists another type of change of attractivity. Suppose that in the spectrum of the Jacobi matrix there exists a pair of complex conjugated eigenvalues which cross an imaginary axes from the left to the right with nonvanishing speed. In this case the trajectories of the system starting in the small neighbourhood of the attracting part of the slow manifold follow it until the point of change of attractivity. But after crossing this point the trajectories do not leave this small neighbourhood immediately. The loss of attractivity is delayed: for some time the trajectories stay near repelling part of the slow manifold and then jump away. This phenomenon was described in [2]. Let us mention that the presence of the additional parameter allow us to change the time of the attractivity loss delay [4].

\section{Ziegler pendulum}

We consider the Ziegler system which describes a double pendulum with elastic hinges under the external force $P$ (fig. 1) [5]: 


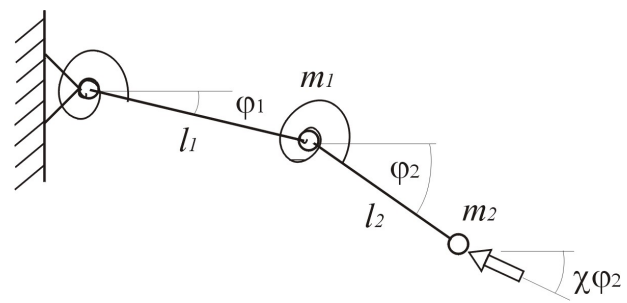

Fig. 1. Ziegler pendulum.

The behaviour of such a system can be described by the Lagrange equations. In the case that the spring rigidity is high, then the system has a big parameter. Suppose that the strength of the force $P$ is slowly changing according to the law $p=p_{0}+\varepsilon \tau$, where $0<\varepsilon \ll 1$. It means that the system becomes multispeed. By using the integral manifold theory we obtain the following system of ordinary differential equations

$$
\begin{gathered}
\dot{p}=\varepsilon, \\
\varphi_{1}=T^{\varepsilon}\left(\varphi_{1}+\kappa\left((1+\mu) \cos \left(\varphi_{1}\right)+\cos \left(\varphi_{2}\right)\right)+\mathrm{p}\left(\sin \left(\chi \varphi_{2}-\varphi_{1}\right)-\sin \left((\chi-1) \varphi_{2}\right)\right)\right) \\
\varphi_{2}=-\varepsilon\left(\varphi_{2}+\kappa\left(\left(2 \cos \left(\varphi_{2}\right)+(1+\mu) \cos \left(\varphi_{1}\right)\right)+\mathrm{p}\left(2 \sin \left((\chi-1) \varphi_{2}\right)-\sin \left(\chi \varphi_{2}-\varphi_{1}\right)\right)\right)\right) .
\end{gathered}
$$

Here $\kappa$ is a small paranteter inversely related to the spring rigidity, $\mu=\mathrm{m}_{1} / m_{2}$. The system possesses the stationary state $\varphi_{1}=\varphi_{2}=0$. Linearization of the fast subsystem near this manifold leads to the form

$$
\begin{gathered}
\dot{p}=\varepsilon \\
\varphi_{1}=-\varepsilon\left(\varphi_{1}(p-1)+\varphi_{2} p(1-2 \chi)\right) \\
\varphi_{2}=-\varepsilon\left(p \varphi_{1}-\varphi_{2}(1+\mathrm{p}(3 \chi-2))\right)
\end{gathered}
$$

The characteristic equations is

$$
(\lambda+1-3 / 2 p(1-\chi))^{2}+\mathrm{p}^{2} / 4(1-\chi)(9 \chi-5)=0 \text {. }
$$

From this, it follows that for $0<\chi<5 / 9$ the last equation has two real eigenvalues, and for $5 / 9<\chi<1$ it has a pair of complex conjugated eigenvalues. In the case of real eigenvalues there exists the point $p_{c r}$ defined by

$$
p_{\text {cr }}=3 / 2-1 / 2 \sqrt{(5-9 \chi) /(1-\chi)}
$$

Such that for $p<p_{c r}$ all eigenvalues are negative, for $p=p_{c r}$ one eigenvalue is zero and another one is negative, for $p>p_{c r}$ one eigenvalue becomes positive. Therefore the slow manifold $\varphi_{1}=\varphi_{2}=0$ is attracting for $p<p_{c r}$, and repelling for $p>p_{c r}$. Therefore the solution $\varphi_{1}=\varphi_{2}=0$ is a canard solution. All other solutions, starting for $p<p_{c r}$ approach the small neighbourhood of the attractive part of the slow manifold and follow it until the point $p=p_{c r}$. After that they follow for some time the repelling part of the slow manifold and then jump away.

In case of complex eigenvalues the value of $p_{c r}$ is defined by the equation

$$
2-3 p(1-\chi)=0 \text {. }
$$

Then the eigenvalues are in the left half plane for all $p<p_{c r}$, for $p=p_{c r}$ the system has a pair of pure imaginary eigenvalues, and for $p>p_{c r}$ the real part of them becomes positive. It means that the solutions of the system possesses delay of loss of attractivity. The trajectories starting at the point with $p<p_{c r}$ approach the attractive part of the slow manifold and follow it until the point $p=p_{c r}$. After that for some time they follow the repelling part of the slow manifold and then jump away.

We note that in both cases as farther the trajectory starts from the change stability point as longer it will follow the repelling part of the slow manifold.

Next pictures show the different solutions to the system for different starting points $p$ and different values of the parameter $\chi$.
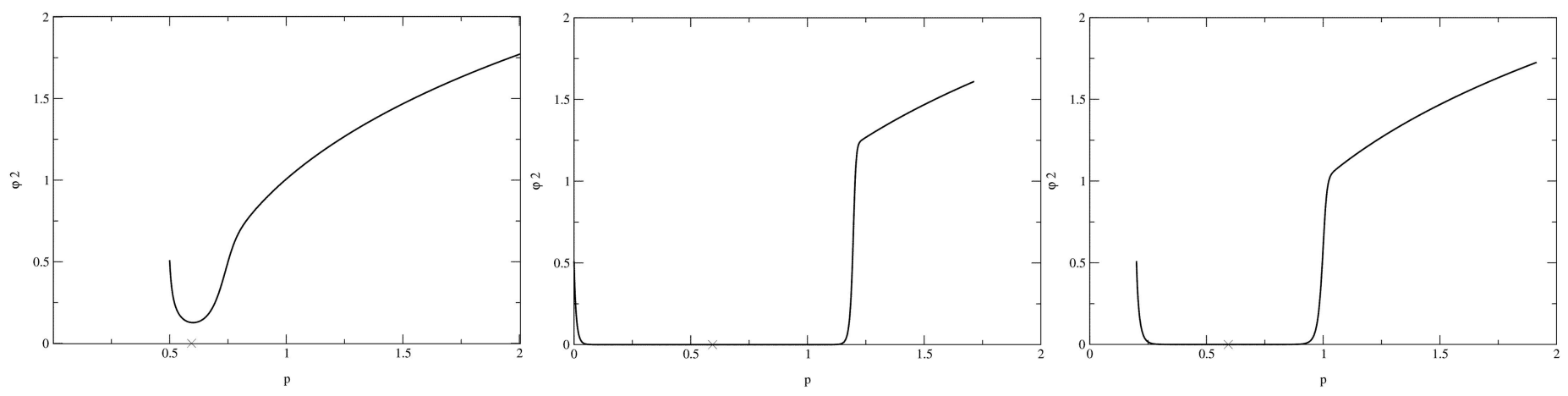

Fig. 2. Canard solutions for different initial value of $p$ in the case $\chi<5 / 9$. 


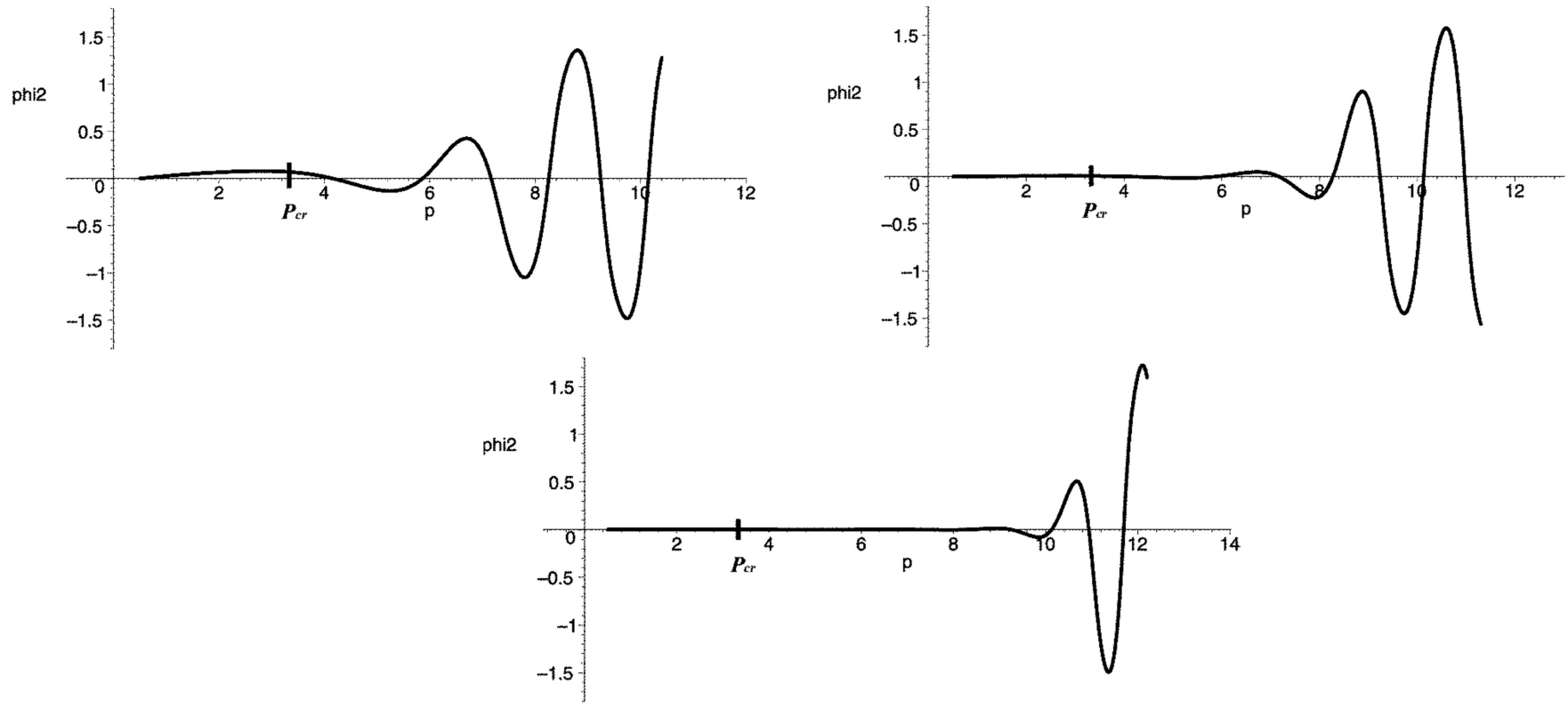

Fig. 3. Solutions with delayed loss of attractivity for the case $\chi>5 / 9$.

\section{References}

[1] Gorelov GN, Sobolev VA, Shchepakina EA. Singularly perturbed combustion models. Samara, 1999; 198 p. (in Russian)

[2] Neishtadt AI. Persistance of stability loss for dynamical bifurcations, I. Differents. Uravn, 1987; 23(12): 2060-2067. II. Differents. Uravn, 1988; 24(2): 226-233. (in Russian)

[3] Strygin VV, Sobolev VA. Separation of motions by the integral manifolds. M.: Nauka, 1988; 256 p. (in Russian)

[4] Shchetinina EV. Integral manifolds for slow-fast systems and delayed loss of stability. Vestnik SGU 2010; 6(80): 93-105. (in Russian)

[5] Ziegler H. Die Stabilitaetskriterien der Elastomechanik. Ingenieur-Archiv, 1952; Band XX: 49-56. 